\title{
Licensed Biologics Manufacturer
}

National Cancer Institute

\section{Source}

National Cancer Institute. Licensed Biologics Manufacturer. NCI Thesaurus. Code

C133299.

A licensed biologics manufacturing facility responsible for production and distribution of medical devices intended for use in the US. 